\title{
EXPLORING FILM DIALOGUE DISCOURSE ${ }^{1}$
}

\author{
Alena Kačmárová
}

\begin{abstract}
The paper falls in the area of discourse analysis and presents an initial framework for research into the nature of audio-visual text, namely the discourse of film dialogue. This preliminary exploration should serve the purpose of finding areas worth further examination. The corpus to be studied is an episode from Friends (2002/2003). The utilized method is a SWOT analysis (an analysis in terms of strengths, weaknesses, opportunities, and threats) within which specific features are indicated and observed. In the paper it is proposed and discussed that the strength of the studied discourse is its being a paragon of neutral/informal speech and idiomaticity of the language; the weakness is seen in the relative 'unnaturalness' of the verbal conduct; the opportunities are represented by their potential to build pragmatic awareness and intercultural competence in viewers; the threat can be embodied in an incautious choice of the title or topic with regard to the viewers' age and intellectual capacity. The explored features are explained and exemplified utilizing portions from the target corpus. The preliminary enquiry triggers further questions and implications.
\end{abstract}

\section{Key words}

film dialogue, sitcom, SWOT analysis, phonetic analysis, sentence structure, verbal behavior

\section{Introduction}

Douglas Biber (in Foreword to Quaglio's “Television Dialogue - The sitcom Friends vs. natural conversation”, 2009, xiii) poses thought-provoking questions:

Don't we talk just like people on television? Or rather, don't those people talk just like us? Conversations on television seem completely natural to the normal viewer. But is that because we have come to expect a particular style of interaction on TV, or because those interactions accurately capture the actual linguistic characteristics of everyday conversation?

Such thinking directs our attention to the question of whether or not (and if so in what aspects) audiovisual text (on the term, cf. Romero Fresco 2009), more specifically a film dialogue, can serve as a model for its viewers' linguistic behaviour. TV discourse has many distinctive features. As viewers we are exposed to informal and vague lexis, loose grammatical structure, expressing personal emotions, narrative features, etc. Moreover, a film dialogue is often 
a reflection of the dynamics of language and conversational routines typical of particular communication acts and roles taken up in the social setting. It also makes available cultural aspects of the target culture. Many aspects are illustrated in the verbal production tagged film dialogue. The initial study has been conducted to specify the areas worth a more complex examination.

\section{Methodology}

The genre selected for the study is a film dialogue of a sitcom. The target discourse is that of the sitcom Friends, specifically the episode The One When No One Proposes (Episode 1, Season IX, 2002/03). Sitcom is typically equated with the USA as the country of origin; for this reason the first choice is the sitcom of American production; one of the most popular sitcoms, as it were also most influential is the sitcom Friends. The choice of the season and episode was governed by the criterion of current usage (post-2000 ones were considered). The aim of the analysis is to identify the language potential of the sitcom dialogue from a viewer's viewpoint. The method chosen for this purpose is articulating pros and cons of its being a relevant model for the linguistic behaviour of its viewers.

My aim is to argue that even though it is not a completely flawless resource, the pros outweigh cons; in doing so my intention is to demonstrate that a film dialogue is an attractive language source with promising capacity for the advancement of one's verbal skills. The tool exercised for this purpose is a SWOT analysis (an analysis in terms of strengths, weaknesses, opportunities, and threats); though typically used in the business context, it is adopted here to describe the discourse of a film dialogue - to present strengths and opportunities on one side, and weaknesses and threats on the other.

The objective is to justify the following claims:

a) The strength of the selected discourse is its being a paragon of neutral/ informal speech and idiomaticity of the language.

b) The opportunities can be anticipated in its potential to build pragmatic awareness and intercultural competence in viewers.

c) The weakness of the selected discourse is in the relative 'unnaturalness' of the verbal conduct.

d) The threat can be posed by an incautious choice of the title or topic with regard to the viewers' age and intellectual capacity.

The discussion will be summed up by concluding remarks. 


\section{The pros of using a film dialogue as a model for linguistic behaviour}

\subsection{Strengths}

\subsubsection{Mistrík's approach to defining oral informal discourse}

Oral forms of communication as such are delineated by certain characteristics. As Halliday (1978: 64) points out, the mere manifestation of the mode is by ".. forms of cohesion, e.g. question-and-answer with the associated type of ellipsis..., the patterns of voice and theme ..., the forms of deixis, e.g. exophoric [situation-referring] the; and the lexical continuity..." [his italics]. These can be associated with orality/vocality, conversationality, familiarity, contextuality, and expressiveness (my translation of Mistrík's terms, cf. 1997: 502-507). Although it is obvious that these features need to be understood as complementing each other, in the present study they will be dealt with piecemeal so that it can be explicitly stated how the target discourse demonstrates the features concerned (cf. Kačmárová 2005); the examples provided originate in the target corpus.

A) Orality/vocality is linked with melody, tempo, rhythm, stress, i.e. the features having a significant share in communicating meaning (Bilá \& Džambová 2010). They truly represent authentic pronunciation varieties. Thus, they are justly the target of imitation by non-native speakers. The study on the duration of intrasentential pauses was carried out on the sample of 50 utterances (altogether 272 pauses) of the target episode (Bilá et al. 2011). The phonetic analysis revealed the highest frequency of extremely short pauses. The results are as follows:

\begin{tabular}{|l|l|}
\hline Pause type & $\%$ \\
\hline Extremely short pause & 75.0 \\
\hline Very short pause & 18.7 \\
\hline Short pause & 3.7 \\
\hline Optimum pause & 2.2 \\
\hline Long pause & 0.4 \\
\hline Very long pause & 0.0 \\
\hline Extremely long pause & 0.0 \\
\hline
\end{tabular}

Table 1: The occurrence of pause types

The study results may be attributable to the fact that informal English utterances are typified by the preference for monosyllabic lexemes (of AngloSaxon origin). Furthermore, English as an analytical language has a low incidence of declined and conjugated forms; grammatical categories tend to be expressed by synsemantic lexemes, which, as a matter of fact, may have impact 
on the occurrence of specific types of pauses. Altogether, this reinforces the idea of preciseness and conciseness being typical features of the target discourse.

B) Conversationality implies the dialogic mode of conversation. It is largely present in the focal discourse and is inherently associated with contactestablishing means, such as: address, evaluation comments, politeness strategies, discourse markers, expressions providing feed-back. The examples from the studied episode are as follows:

address

evaluation comments

politeness strategies

discourse markers
Joey: And Ross, I need to talk to you.

Chandler: Oh, God. Please.

Rachel: I'm sorry, honey, what were you saying?

Phoebe: You know, Ross, doctors are supposed to be smart.

feed-back provided Ross: Yeah, right.

C) Familiarity is linked with the private setting tolerating lexical and grammatical slips. The focal discourse presents a party of six main characters involved in a variety of topics on interpersonal (almost intimate) issues. Typically, an episode has three main topics and approximately three support topics. The vocabulary ranges from informal to colloquial; a certain amount of slang is present, too. In terms of grammar, standard and/or conventionalized constructions are in the majority. This discourse is primarily meant to present standard language and is intended as public discourse; hence, grammatical slips tend to be avoided.

D) Contextuality of the discourse entails the participants' awareness of the situation and the issues talked over. It is clearly exhibited by participants' relevant contributions, e.g.:

Rachel: $\quad$ Joey asked me to marry him.

Monica: $\quad$ What?

Rachel: $\quad$ Joey proposed to me.

Monica: Is he crazy?

Sharing situational context and/or background information makes it possible to express relevant contribution, in this case, Monica's astonishment over Rachel's revelation.

Moreover, the involvement of the speaker and the shared knowledge allow for successful interpreting in the case of elliptical structures or deixis, e.g.:

ellipsis

Chandler: Why, why, why would you [...]?

deixis

Rachel: Could you be a dear and go tell him? 
Contextuality implies situational context and extra-linguistic and paralinguistic means. In real conversation situational context is very helpful in interpreting communication intention. In the focal discourse, not only is it a means of interpreting the message but also a source of amusement. It assists in carrying out semantic transfer and it often outweighs verbal conduct. In a way, it replaces complex sentence structures (which would be ineffective and boring), cf. below (2.1.2). Even so, extra-linguistic and paralinguistic means are not further studied in this paper since the focus of the paper is verbal performance.

E) Expressiveness mirrors a speaker's stance to the idea communicated. Common means of its expression are diminutives, augmentatives, interjections, and emphatic expressions. In the focal discourse, expressive language means appear in a moderate number. They are substituted by non-verbal means (face expressions, gestures) and paradoxical situations.

The five features capture the essence of a private, spontaneous conversation and demonstrate idiomatic verbal performance. The focal corpus seems to be a relevant source for contemplation of the linguistic behaviour of native speakers. A film dialogue, a type of audio-visual text is created with ambition to give the illusion of natural/idiomatic linguistic and non-linguistic behaviour. Conversation (largely present in the studied discourse) is the number one source of linguistic and non-linguistic behaviour contemplation. The rhetorical mode of the focal discourse echoes the verbal performance traceable to oral communication. The setting of the selected discourse is the environment shared by friends and family members. Features typical of such an environment are a dialogic mode of speech, frequent turn change and change of speakers, spontaneity (though pretended), expressive intonation, rapid speech tempo, and slightly exaggerated nonverbal communication (at least from a viewpoint of a Slovak speaker of English).

\subsubsection{Syntactic analysis}

The sentence structure greatly influences the overall impression of how demanding the target audiovisual text is in terms of comprehension. For this reason, an analysis of the sentence structure of the chosen episode was conducted. The syntactic analysis aims to unveil how big a challenge the target discourse is for speakers of English. The syntactic level presents a complex issue; it encompasses several areas - from phrases through clauses to clause complexes. The smallest unit on a sentence level is a simple sentence (clause). Simple sentences can be classified based on several criteria, namely according to: a) the number of finite verb forms, b) grammatical structure, and c) the presence and/or absence of secondary sentence elements (cf. Bilá et al. 2011). 
The number of finite verb forms is decisive in the discrimination between simple sentences and clause complexes, which implies the classification of sentences as simple, compound, complex and compound-complex. The criterion of grammatical structure implies the classification of simple sentences into twomember and one-member sentences, based on whether both subject and predicate are present, or whether the sentence is composed of only one of the two sentence elements; the sentence is considered two-member even if the subject is not overtly present, yet implied, e.g. in the case of the English imperative. One-member sentence can be composed of a noun, an adjective, an adverb, an interjection, or an address form. Sentences are classified as extended or non-extended based on whether a sentence is composed of both primary and secondary or just of primary sentence elements respectively (Nižníková 1994, Rafajlovičová 2003).

The utterances of the chosen episode were classified based on the three criteria mentioned above: 1) the number of finite verb forms, 2) grammatical structure, and 3) presence or absence of secondary sentence elements. The syntactic analysis was conducted within three dichotomous pairs: 1) simple sentences vs. clause complexes, 2) one-member sentences vs. two-member sentences, and 3) nonextended sentences vs. extended sentences. The sentences were analyzed as syntactic constructs. It is necessary to point out the difference between a sentence and an utterance. Hereinafter, the term 'utterance' will represent situationally and contextually determined realization of a speaker's communication intention; the term 'sentence' will denote an autonomous structure (predicative or nonpredicative) semantically complete, with a certain intonation pattern and grammatically ordered elements. (Oravec \& Bajzíková 1986: 46-48)

In the chosen episode, based on the above delineation of a sentence, 507 sentences as syntactic constructs were identified and classified within the three dichotomous pairs. In the first one, the discrimination between simple sentences and clause complexes (of any kind, whether compound, complex, or compoundcomplex) was considered. In the studied corpus, simple sentences significantly outnumbered clause complexes. Out of 507 sentences, 399 were simple sentences (78.70\%), 108 were clause complexes $(21.30 \%)$, cf. Graph 1.

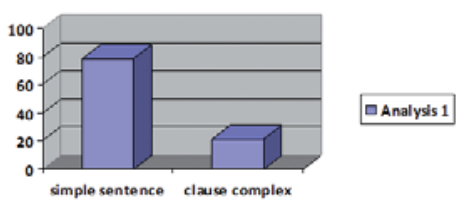

Graph 1: The occurrence of simple sentences and clause complexes expressed in percentage 
The second step was the classification of sentences according to the grammar structure, which is to say discrimination between one-member and two-member sentences (as explained above). In this dichotomous pair, the total number of sentences is higher than in the first one since clause complexes were divided into clauses and each clause was treated separately. In order to calculate the frequency, the total number of clauses considered was 651 . Out of this number, 115 were one-member $(17.67 \%)$ and 536 were two-member sentences/clauses $(82.33 \%)$, cf. Graph 2.

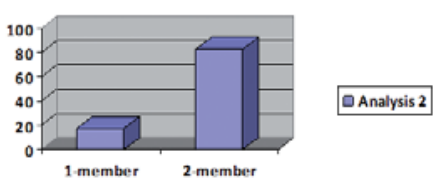

Graph 2: The occurrence of one-member and two-member sentences expressed in percentage

The third step was the classification of sentences based on what sentence elements were present. The total number of sentences was identical with that in the second dichotomous pair, i.e. 651 . The clauses were considered as nonextended if only the subject and predicate were present; the clauses were considered as extended if secondary sentence elements were present, too. Out of 651 sentences, 335 clauses were non-extended (51.46\%), while 316 clauses were extended (48.54\%), cf. Graph 3.

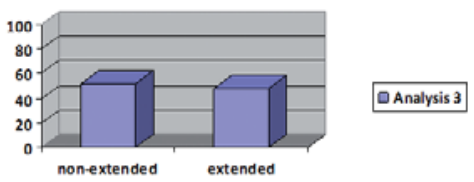

Graph 3: The occurrence of non-extended and extended sentences expressed in percentage

The syntactic analysis of the utterances in the chosen episode indicated the prevalence of simple sentences (SS) over clause complexes (CC), the prevalence of two-member sentences (2MS) over one-member sentences (1MS) and a slightly higher number of non-extended sentences (NES) over extended sentences (ES).

The analysis indicated a high ratio of simple sentences vs. a very low ratio of clause complexes. In the dichotomous pair one-member vs. two-member sentences, two-member sentences prevailed; however, due to the size of the corpus the occurrence of one-member sentences can be considered relatively 
high. The juxtaposition of non-extended and extended sentences reveals a high ratio of non-extended sentences, almost comparable with that of extended sentences. Drawing on anecdotal evidence, it can be assumed that such sentence structure adds to the attractiveness of the target discourse when considered a model in linguistic behaviour.

\subsection{Opportunities}

The opportunities that can be seen in the exposure to authentic audiovisual text are direct contact with certain extra-linguistic reality and familiarization with cultural values and meanings, which enhances intercultural competence; this can be justified as follows. The chosen discourse presents a way of life and social (and other) values that are an intrinsic part of a particular society and/or culture and of a period. These values and meanings are mediated to the viewers; in this way viewers can confront what they see and observe not only with their own habits, beliefs and opinions but also with what they know about the target culture, about the established stereotypes. Stereotyping is usually assigned negative connotation, because if a particular feature is, though unknowingly or not on purpose, applied to the whole nation en bloc, it boosts the building of bias. However, stereotypes can serve a particular purpose, especially in one's endeavour to understand behaviour and communication strategies of a particular culture.

In an effort to define culture, one has to realize its multidisciplinary nature and its being a complex phenomenon. The widespread approach is the recognition of broad and narrow senses of culture. The narrow sense implies art, music, literature, food, or dress; the broad sense entails the shared background (national, ethnic, or religious), which is to say customs, beliefs, attitudes, values on the one hand, and common language and a communication style on the other. Succinctly, "[c]ulture is a social phenomenon that tells us what we should do and how we should behave" (Kominarec \& Kominarecová 2009: 13; my translation). I will adhere to the view that culture is a community within which people share social and historical background and prior experience with lexical units and utterances, and behave according to conventionalized linguistic and non-linguistic patterns.

The chosen sitcom makes available certain verbal conduct, a view of life and the mindset of an American society, in particular the area of New England. For example, the utterance "Doctors are supposed to be smart" implies American respect towards the academic title Ph.D.; the utterance "...Mr. Hazmegian still has my Game boy" hints an idea of multiculturalism and tolerance towards other cultures. In the episode under study, the theme is an imaginary engagement, which foregrounds some typical cultural streaks: the American passion for throwing parties, an inclination to share engagement celebrations with friends and family, 
efforts to think of the most original ways of proposing, and the importance of the size of the diamond in an engagement ring. In the whole episode, American informality is clearly depicted. The most obvious situation is greeting a person; in the target episode, only two greetings are used: "Hey" and "Hi". Another means of expressing informality is short answers with no pro-forms extensively present (e.g. "No" instead of "No, I don't").

A conscientious user of a foreign language realizes that every language is unique in its own way - it is unique in how people perceive the extralinguistic reality, label extralinguistic objects, interpret what they see and hear, construct ideas, or infer meanings. This is due to the idiosyncratic nature of every single culture. Only if we are aware of culturally determined discrepancies between native and foreign languages, can we acquire competence that is beyond the knowledge of a language and helps eliminate apprehension of social encounters in an authentic setting. Such knowledge includes those aspects of culture that assist in acquiring the conversation style and conventionalized routine of a language. Language and culture are complementary to each other, hence inseparable.

\section{Cons of using a film dialogue as a model for linguistic behaviour}

\subsection{Weaknesses}

The discourse under discussion also has a weak point - the supposed unnaturalness of the verbal conduct. The conversation concerned may seem unnatural for several reasons. Firstly, the dialogue build-up follows a particular goal - to make people laugh. Hence, it seems that the characters are always alert and in whatever situation ready to contribute a meaningful, purposeful, but most of all witty remark. In real life, we are never funny all the time. For this reason such conversation may be viewed as 'artificial' (in the sense of stylized) and not illustrating authentic verbal conduct. Secondly, the focal communication is not spontaneous. It is primarily controlled by a scriptwriter, a producer, and an actor. The focal discourse is natural speech transformed to the media text. It can be referred to as hybrid communication, somewhat "arranged spontaneous speech" (cf. Bilá et al. 2011). It is text presenting the illusion of spontaneous turn taking. The conversation is carefully planned and revised so that it looks like spontaneous dialogue-based communication. Even so, a common language user, and/or viewer, perceives audio-visual text as authentic spoken utterance. A film dialogue has an important function - it helps the story develop, it assists in depicting characters and makes available social values of mother culture. 


\subsection{Threats}

In the application of the SWOT analysis, the last element is bearing in mind possible threats. In principle, the analyzed discourse does not pose a threat to a language user. In the sitcom under discussion, episodes disclose stories and personalities of intellectual Ross, spoiled Rachel, sarcastic Chandler, competitive Monica, eccentric Phoebe and childish Joey. The sitcom illustrates the value of friendship in diverse and humorous ways, and so offers solutions to everyday problems. However, when considering a film dialogue as a source of imitation, attention needs to be paid to the choice of the title, episode, or topic with regard to the profile of a viewer, including their age and intellectual capacity. It is not only the sitcom Friends but also other titles that present a friendly atmosphere and personal to intimate topics with a certain amount of age-determined sensitive issues and slang related to it. Careful selection may help avoid embarrassing situations or inappropriateness of the vocabulary. We need to consider whether or not viewers are mature enough to be exposed to what life brings to particular characters in a particular episode of a particular title.

\section{Conclusion}

The target corpus was studied with the aim to point to the pros and cons of the sitcom Friends discourse. The tool utilized was the SWOT analysis - pros were represented by strengths and opportunities, cons by weaknesses and threats of utilizing a film dialogue (in a sitcom genre) for the purposes of language study. By means of the observation of linguistic behaviour, and through phonetic and syntactic analyses I aimed to justify claims that the target discourse is a nice example of neutral/informal speech and of the idiomaticity of the language, and that it has good potential to build pragmatic awareness and intercultural competence in viewers in spite of its relative (no, substantial) lack of authenticity and/or spontaneity, and despite the necessity to be cautious in the choice of a title and/or overall topic for use in class.

The implications generated by the initial exploration may as well steer our attention in several directions. As it were, the underlying reason for attendance to conversation techniques of the second language may well be getting insight into the mindset of native speakers in order to provide ways of capturing the idiomaticity of a foreign language (on idiomaticity, cf. Kavka 1999), which is to say preferred ways of saying things and of organizing thoughts (cf. Kecskes 2007). It follows that culture serves as a means of understanding how language operates. However, a language can also serve as a guide to understanding basic assets and social values of a particular culture. Favouring a particular lexical item 
or utterance over others in a certain speech event mirrors a speaker's command of pragmatic aptness. Tárnyiková (2000: 307) points out, "there is a generally shared consensus that while native speakers are more tolerant of grammatical mistakes, pragmatic failure is very often regarded as intentionally impolite or offending."

The implications for linguistics practice are such that research on the sitcom discourse has great potential and provides a rich source of language material for exploring phonetic features, sentence structure, discourse markers, language formulae, or pragmatic interpretation of semantic features. Hence, further research can focus on a specific linguistic issue (in a larger corpus of language data), the idiolect of a particular character, or the idiomatic execution of a particular illocutionary act on phonetic, morphosyntactic, lexical, or stylistic language levels while considering pragmatic appropriateness of the communicative act. In doing so, the correlation between social norms and language means employed in the make-up of a speech event can be arrived at.

\section{Endnote}

1 The research was conducted within the author's research activities in the project "Retrofitting and Extension of the Center of Excellence for Linguaculturology, Translation and Interpreting", supported by the Research \& Development Operational Programme funded by the ERDF. Project code: 26220120044, Agreement Number: 031/2009/2.1/OPVaV.

\section{References}

Bilá, M. and Džambová, A. (2010) Intonačná štruktúra vety. Porovnávací aspekt. Prešov: PULIB.

Bilá, M., Džambová, A. and Kačmárová, A. (2011) Fonetické, syntaktické a pragmatické aspekty hovoreného prejavu. Prešov: Faculty of Arts. University of Prešov.

Halliday, M. A. K. (1978) Language as Social Semiotic. London: Edward Arnold.

Kačmárová, A. (2005) 'Internet chatting inside out.' In: SKASE Journal Of Theoretical Linguistics, Vol. 2, No 1 SKASE \& PULIB, Prešov. Online document. 1 September 2012. <http://www.skase.sk/ Volumes/JTL02/index.htm>.

Kavka, S. (1999) 'Some aspects of idiomaticity.' In: Proceedings of the Conference "European and American Studies at the turn of the Millennium". Ostrava: University of Ostrava. 101-104.

Kecskes, I. (2007) 'Formulaic language in English Lingua Franca.' In: Kecskes, I. and Horn, L. (eds) Explorations in Pragmatics: Linguistic, Cognitive and Intercultural Aspects. Berlin/New York: Mouton de Gruyter. 191-219.

Kominarec, I. and Kominarecová, E. (2009) Multikulturalita a komunikácia. Prešov: Prešovská univerzita.

Mistrík, J. (1997) Štylistika. Bratislava: SPN.

Nižníková, J. (1994) Praktická príručka slovenskej skladby. Prešov: Slovakontakt.

Oravec, J. and Bajzíková, E. (1986) Súčasný spisovný slovenský jazyk. Syntax. Bratislava: SPN. 
Quaglio, P. (2009) Television Dialogue - The Sitcom Friends vs. Natural Conversation. Amsterdam/Philadelphia: John Benjamins.

Rafajlovičová, R. (2003) A Survey of the English Syntax. Prešov: PU.

Romero Fresco, P. (2009) 'A corpus-based study on the naturalness of the Spanish dubbing language: The analysis of discourse markers in the dubbed translation of friends.' Online document. 7 May $2014<$ http://www.ros.hw.ac.uk/bitstream/10399/2237/1/ RomeroFrescoP_0209_sml.pdf $>$

Tárnyiková, J. (2000) 'Pragmatics.' In: Štekauer, P. (ed.) Rudiments of English Linguistics. Prešov: Slovacontact. 271-312. 